\title{
Model-assisted time-synchronization of cardiac MR image and catheter pressure data
}

\author{
Maria Gusseva ${ }^{1,2}[0000-0002-9024-7829]$, Joshua S. Greer ${ }^{3}$, Daniel A. \\ Castellanos $^{4[0000-0003-1188-5257]}$, Mohamed Abdelghafar Hussein ${ }^{3,5}$, Gerald \\ Greil $^{3}$, Surendranath R. Veeram Reddy ${ }^{3}$, Tarique \\ Hussain $^{3[0000-0003-4091-992 X]}$, Dominique Chapelle ${ }^{1,2}$, and Radomír \\ Chabiniok $^{1,2,3[0000-0002-7527-2751]}$ \\ 1 Inria, Palaiseau, France \\ 2 Laboratoire de Mécanique des Solides (LMS), Ecole Polytechnique/CNRS/Institut \\ Polytechnique de Paris, Palaiseau, France \\ 3 Division of Pediatric Cardiology, Department of Pediatrics, UT Southwestern \\ Medical Center, Dallas, TX, USA \\ 4 Department of Cardiology, Boston Children's Hospital, Boston, MA, USA \\ ${ }_{5}$ Pediatric department, Kafrelsheikh University, Kafr Elsheikh, Egypt \\ radomir.chabiniok@utsouthwestern.edu
}

\begin{abstract}
When combining cardiovascular magnetic resonance imaging (CMR) with pressure catheter measurements, the acquired image and pressure data need to be synchronized in time. The time offset between the image and pressure data depends on a number of factors, such as the type and settings of the MR sequence, duration and shape of QRS complex or the type of catheter, and cannot be typically estimated beforehand. In the present work we propose using a biophysical heart model to synchronize the left ventricular $(\mathrm{LV})$ pressure and volume $(\mathrm{P}-$ $\mathrm{V})$ data. Ten patients, who underwent CMR and LV catheterization, were included. A biophysical model of reduced geometrical complexity with physiologically substantiated timing of each phase of the cardiac cycle was first adjusted to individual patients using basic morphological and functional indicators. The pressure and volume waveforms simulated by the patient-specific models were then used as templates to detect the time offset between the acquired ventricular pressure and volume waveforms. Time-varying ventricular elastance was derived from clinical data both as originally acquired as well as when time-synchronized, and normalized with respect to end-systolic time and maximum elastance value $\left(E_{\text {orig }}^{N}(t), E_{\mathrm{t}-\mathrm{syn}}^{N}(t)\right.$, respectively). $E_{\mathrm{t}-\mathrm{syn}}^{N}(t)$ was significantly closer to the experimentally obtained $E_{\exp }^{N}(t)$ published in the literature $\left(\mathrm{p}<0.05, L^{2}\right.$ norm). The work concludes that the model-driven time-synchronization of P-V data obtained by catheter measurement and CMR allows to generate high quality $\mathrm{P}-\mathrm{V}$ loops, which can then be used for clinical interpretation.
\end{abstract}

Keywords: interventional cardiovascular magnetic resonance imaging . time-synchronization of clinical data $\cdot$ cardiovascular modeling $\cdot$ pressurevolume loops $\cdot$ personalized medicine $\cdot$ translational research 


\section{Introduction}

The analysis of intraventricular pressure-volume (P-V) loops contributes to the detailed assessment of the heart function and can be employed when planning a complex intervention. Combining the catheter pressure measurement with cardiovascular magnetic resonance imaging (CMR) yields rich morphological and functional datasets of the current state of patients' pathology. However, the recorded ventricular pressures and volumes are often not precisely synchronous, even during a simultaneous acquisition, which affects the shape of the P-V loop (see Fig. 1). There are three principal reasons for this P-V dyssynchrony:

1. Data of cine MRI are obtained over a number of cardiac cycles and the reconstructed time-volume plot therefore represents an average cycle during the acquisition.

2. The detection of R-peak in the strong magnetic field of MRI is known to be challenging, particularly in patients with a pathological QRS complex in whom the $\mathrm{S}$ wave or $\mathrm{R}$ ' wave (e.g. in some right bundle branch block patients) may be detected instead. This leads into the time offset up to the QRS duration.

3. The fluid-filled catheter is known to record the pressure changes with some delay, which varies depending e.g. on the catheter size.

The time offset caused by these points varies among the patients and practically cannot be predicted beforehand. In this work we propose to create biophysical models of a reduced complexity using the patient-specific information of the maximum and minimum ventricular volume and pressure provided by the CMR and catheter data. These models will then be used as a template for the timesynchronization of the measured P-V data (see the pipeline depicted in Fig. 2). Qualitative assessment will be carried out by plotting the P-V loops using the original and the time-synchronized pressure and volume waveforms. The quantitative comparison will be performed by comparing the generated ventricular time-varying elastance functions with the experimentally obtained time-varying elastance published by Suga et al. [15]. Furthermore, some functional characteristics of the cardiovascular system not directly visible in the data can be obtained thanks to using a patient-specific biophysical model.

\section{Methods}

\subsection{Data}

Datasets of left ventricles of ten patients with repaired tetralogy of Fallot (rTOF) were included in the study. The data collections were performed under the ethical approvals of the Institutional Review Board of UT Southwestern Medical Center Dallas (STU-2020-0023). The IRB waived the need for a consent to use the anonymized retrospective data. Cine MRI data with retrospective ECG gating (with parallel factor SENSE=2, temporal resolution $\sim 30 \mathrm{~ms}$ ) covering both 


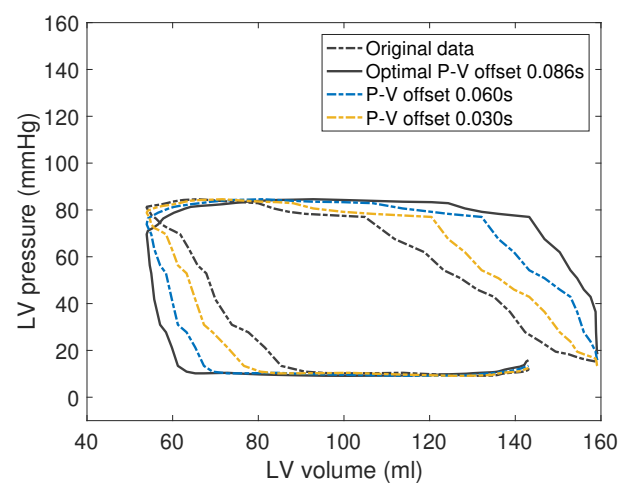

Fig. 1. Illustration of the pressure-volume (P-V) offsets on the shape of the P-V loop in the measured data.

ventricles and phase-contrast flow through the aortic valve were acquired. The ventricular volumes from cine MRI were obtained using the CVI42 software (Circle Cardiovascular Imaging Inc., Calgary, Canada) combined with the motion tracking algorithm of [4]. The catherization was performed in a separate session after CMR. A left-heart cathether was advanced into aorta and left ventricle, where the pressures were recorded during a breath-hold.

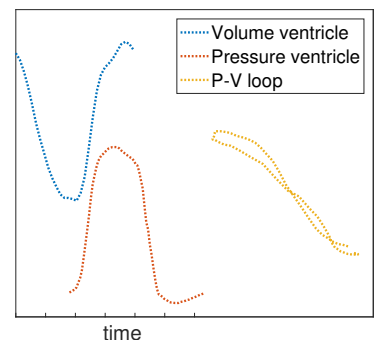

(a) Original data

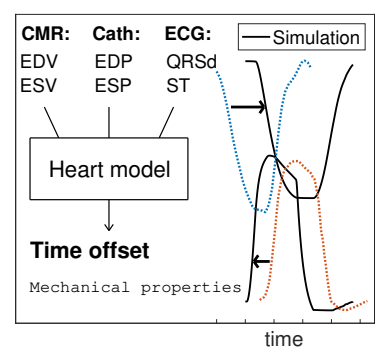

(b) Patient-specific model

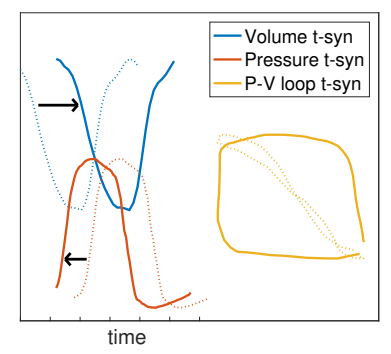

(c) Time-synchronizion

Fig. 2. Time-synchronization pipeline. (a) Original pressure-volume (P-V) data; (b) Patient-specific time offset detected by the model (black arrows); (c) Time offset detected in (b) applied on original P-V data in (a) to obtain time-synchronized (t-syn) $\mathrm{P}-\mathrm{V}$ data (solid lines). ED/ESV: end-diastolic/-systolic volumes (ventricle); ED/ESP: end-diastolic/-systolic pressures (ventricle); ECG: electrocardiogram timings.

\subsection{Biophysical heart model}

A reduced-complexity biomechanical model of left ventricle (LV) and systemic circulation was employed [1]. The schematics of the model is depicted in Fig. 3. 
The geometry and kinematics of the ventricle are reduced to a sphere with an inner radius $\mathrm{R}$ and a wall thickness $\mathrm{d}$, while the constitutive mechanical laws are preserved as in the full 3D heart model $[3,12]$. The mechanical behavior of the myocardium is described by a rheological model of Hill-Maxwell type [5] that contains an active contractile component (actin-myosin complex) and a viscoelastic component (collagen-rich elements in the surrounding connective tissue). The active component is represented within Huxley's sliding filament theory $[6,7]$. All myosin filaments in the sarcomere unit are considered as a series of springs, with active stress $\left(\tau_{c}\right)$ and active stiffness $\left(k_{c}\right)$ produced for sarcomere extension $\left(e_{\text {fib }}\right)$ given by, see [3]:

$$
\left\{\begin{array}{l}
\dot{k}_{c}=-\left(|u|+\alpha\left|\dot{e}_{\mathrm{fi}}\right|\right) k_{c}+n_{0}\left(e_{\mathrm{fib}}\right) k_{0}|u|_{+} \\
\dot{\tau}_{c}=-\left(|u|+\alpha\left|\dot{e}_{\mathrm{fib}}\right|\right) \tau_{c}+\dot{e}_{\mathrm{fib}} k_{c}+n_{0}\left(e_{\mathrm{fib}}\right) \sigma_{0}|u|_{+},
\end{array}\right.
$$

where $u(t)$ is an electrical activation function representing intracellular calcium kinetics that induces contraction (when $u>0$ ) or relaxation $(u<0)$ of the myocardium (Fig. 3b). The parameter $\alpha$ is a bridge destruction rate upon rapid change in length in the sarcomere [3]. The parameter $\sigma_{0}$ is the active stress developed by the sarcomere during systole under optimal extension $e_{\mathrm{fib}}$, and $n_{0}\left(e_{\mathrm{fib}}\right) \in[0,1]$ represents the Frank-Starling law [3]. The parameter $\sigma_{0}$ will be further referred to as the contractility.

The model was turned into patient-specific regime according to the sequential calibration procedure described in [10]. Briefly, the 2-stage Windkessel model was adjusted by imposing the flow measured by phase-contrast MRI aiming to match the maximum and minimum aortic pressure and the pressure at the dicrotic notch. Then, the LV wall thickness and the ventricular volume were prescribed according to the cine MRI. The model stress-free configuration was assumed as in the experimental data of [8]. The ventricular preload was prescribed from the measured end-diastolic ventricular pressure. Passive myocardial properties were calibrated to match the measured end-diastolic volume under imposed preload. Myocardial contractility was adjusted to match the measured stroke volume. Patient-specific parameters adjusted during this process, therefore were in the Windkessel model: distal and proximal resistances of the circulation, distal capacitance of the circulation; in the heart model: myocardial stiffness and contractility. Ventricular stroke work was calculated as the area encompassed within the model-derived P-V loop.

\subsection{Time-synchronization}

Thanks to the biophysical and physiological character of the model, the timing of the cardiac phases was considered as a reference. The simulated waveforms were therefore used as templates to time-synchronize the original data to find an optimal time offset $t_{\text {offset }}$ between the measured data and the model, minimizing the criterion:

$$
\min _{t_{\text {offset }}} \int_{0}^{t_{\mathrm{ES}}}\left(f(t)-g\left(t-t_{\mathrm{offset}}\right)\right)^{2} d t
$$




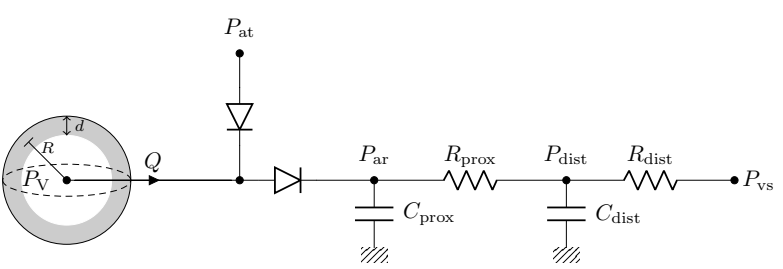

(a) Biomechanical model

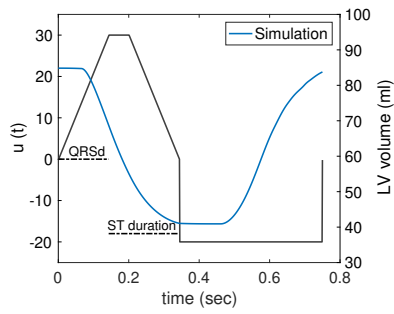

(b) Activation function

Fig. 3. (a) Coupling of the heart with atrioventricular and arterial valves via system of diodes and with circulation system represented by a two-stage Windkessel model. $P_{a t}, P_{V} P_{a r}, P_{d i s t}, P_{v s}$ stand for pressures in left atrium, left ventricle, aorta, distal systemic circulation and venous system; $R_{\text {prox }}, C_{\text {prox }}, R_{\text {dist }}, C_{d i s t}$ are proximal and distal resistances and capacitances in Windkessel model; (b) Electrical activation function $u(t)$ (black line) with imposed timings of duration of QRS and ST segment from electrocardiogram measurements.

where $f(t)$ represents the simulated LV pressure or volume; $g(t)$ represents the measured ventricular pressure (volume); $t_{\mathrm{ES}}$ is the time of end-systole in the data.

The P-V loops were constructed by using both original and time-synchronized waveforms. Time-varying elastance was computed for the original and timesynchronized data as:

$$
E_{\text {orig } / \mathrm{t}-\text { syn }}(t)=\frac{P_{\text {orig } / \mathrm{t}-\text { syn }}(t)}{V_{\text {orig } / \mathrm{t}-\mathrm{syn}}(t)},
$$

where $P_{\text {orig/t-syn }}(t), V_{\text {orig/t-syn }}(t)$ are LV pressures and volumes as originally measured / time-synchronized. The time-varying elastances were then doublenormalized in time (with respect to the end-systolic time $t_{\mathrm{ES}}$ ) and with respect to the maximal elastance value, as in Suga et al. [15]:

$$
E^{N}(\bar{t})=\frac{E\left(\bar{t} \cdot t_{\mathrm{ES}}\right)}{\max (E)} .
$$

\subsection{Statistical analysis}

The distances between the normalized time-varying elastance obtained from the original, time-synchronized data, respectively $\left(E_{\mathrm{orig}}^{N}, E_{\mathrm{t}-\mathrm{syn}}^{N}\right.$, respectively) and the experimentally obtained $E_{\exp }^{N}$ (by Suga et al. [15]) were compared using the $L^{2}$ norm over the whole cardiac cycle, i.e. relative distance RD is given by

$$
\operatorname{RD}\left(E_{\text {orig/t-syn }}^{N}, E_{\text {exp }}^{N}\right)=\frac{\left\|E_{\text {orig/t-syn }}^{N}-E_{\text {exp }}^{N}\right\|_{L^{2}}}{\left\|E_{\text {exp }}^{N}\right\|_{L^{2}}} .
$$

Wilcoxon signed-rank tests at $\mathrm{p}<0.05$ were conducted to assess the difference between $\operatorname{RD}\left(E_{\text {orig }}^{N}, E_{\exp }^{N}\right)$ and $\operatorname{RD}\left(E_{\mathrm{t}-\mathrm{syn}}^{N}, E_{\exp }^{N}\right)$. 


\section{Results}

Fig. 4 shows an example of the patient-specific model and the model-based time-synchronization for Patient \#6. Fig. 5 displays P-V loops for selected patients, while using original or time-synchronized pressure and volume waveforms. Fig. 6(a) shows for patient \#6 $E_{\text {orig/t-syn }}^{N}(\bar{t}$ ) (original and time-synchronized data, respectively), in comparison with the experimentally obtained $E_{\exp }^{N}(\bar{t})$. Median $\operatorname{RD}\left(E_{\text {orig }}^{N}, E_{\text {exp }}^{N}\right)$ and $\operatorname{RD}\left(E_{\mathrm{t}-s y n}^{N}, E_{\text {exp }}^{N}\right)$ (original and time-synchronized data for all patients) were 0.16 and 0.03, respectively, as shown in Fig. 6(b). According to the Wilcoxon signed-rank test, $E_{t-s y n}^{N}(\bar{t})$ was significantly closer to $E_{\exp }^{N}(\bar{t})$ $(\mathrm{p}<0.05)$. Table 1 shows the time offsets for all patients detected by the proposed procedure between original and time-synchronized data. Table 1 (right columns) shows examples of patient-specific quantities derived by the model characterizing the functional state of the heart and circulation system.
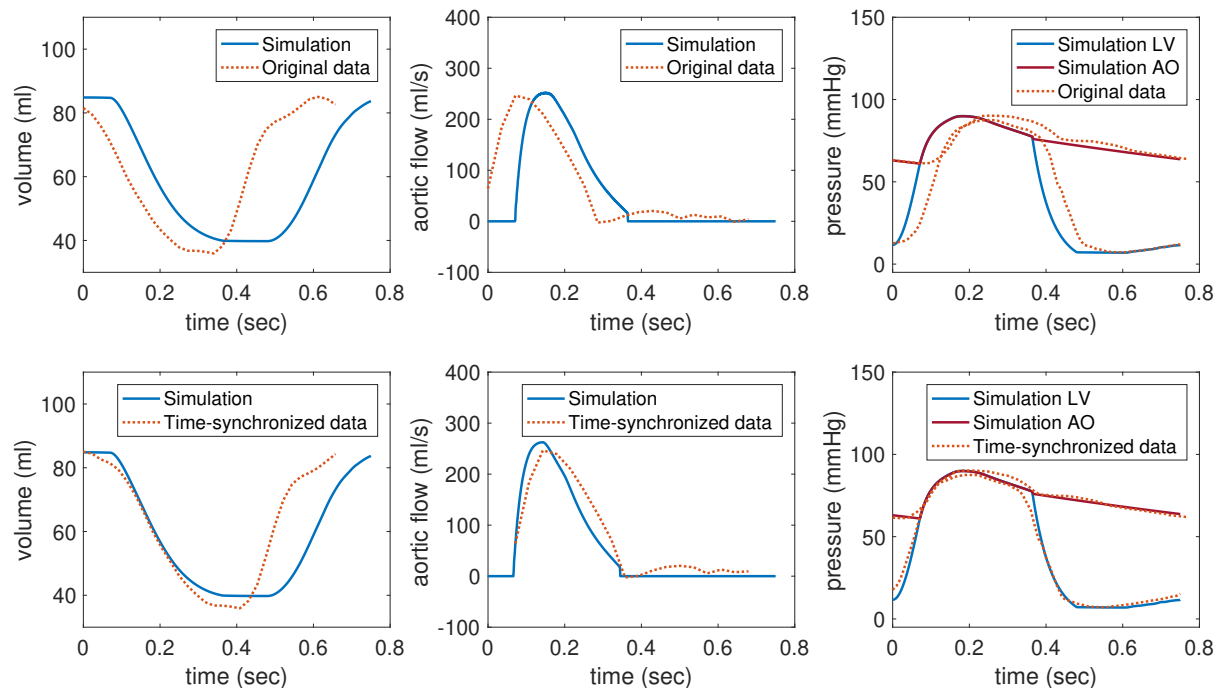

Fig. 4. Example of data-model coupling for Patient \#6. Top: model calibration versus original (non-synchronized) data; bottom: model calibration versus time-synchronized data.

\section{Discussion}

In this work we developed a time-synchronization protocol for the ventricular pressure obtained by catheter measurement and volume from cine MRI using a patient-specific computational model. Fig. 5 shows a qualitative improvement of the $\mathrm{P}-\mathrm{V}$ loops after time-synchronizing the pressure and volume data. The 

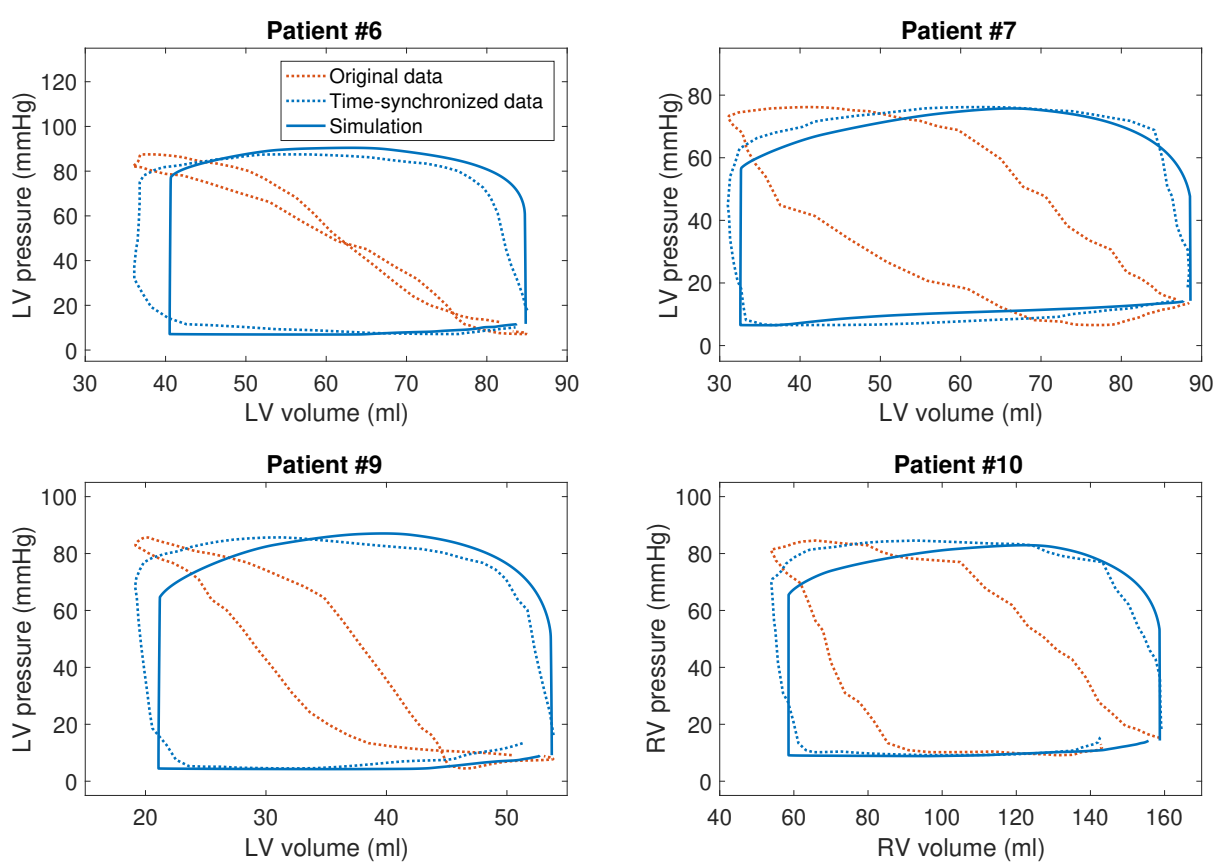

Fig. 5. Pressure-volume loops for selected patients.

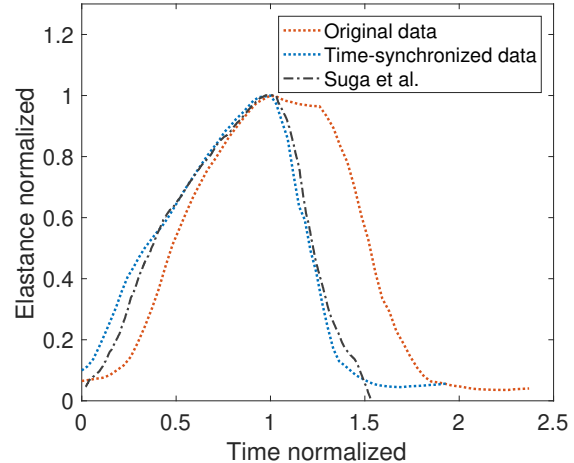

(a) Normalized elastance Patient \#6

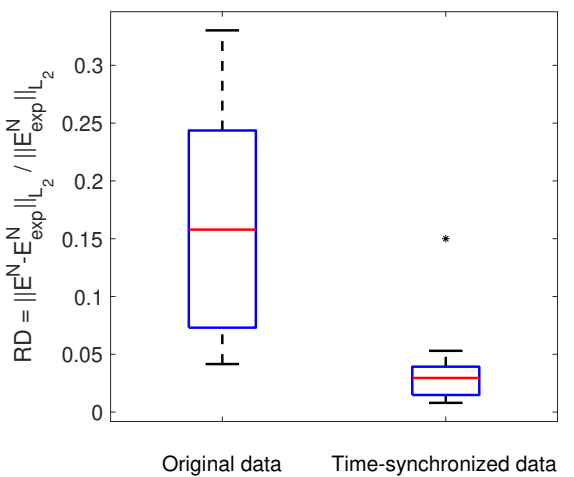

(b) Relative distance all patients

Fig. 6. (a) Normalized elastance for a selected patient versus elastance in [15] (b) Boxplots of relative distance (RD) of normalized elastance between original/timesynchronized data and the elastance in [15] for all patients. Central line inside the boxes indicates the median, and the bottom and top edges of the boxes show the 25th and 75 th percentiles, respectively. The star indicates significant difference at $\mathrm{p}<0.05$. 
Table 1. Model-derived time offsets and biophysical parameters. QRSd: QRS duration; MRI: magnetic resonance imaging; Cath: catherization; $R_{\text {dist }}$ : distal resistance of the circulation.

\begin{tabular}{|l|c|c|c||c||c|c|c|}
\hline & \multicolumn{2}{|c|}{} & Heart rate & Time offsets & \multicolumn{3}{|c|}{ Mechanical indicators } \\
\hline & $\begin{array}{c}\text { QRSd } \\
\mathrm{s}\end{array}$ & $\begin{array}{c}\text { MRI } \\
\text { bpm }\end{array}$ & $\begin{array}{c}\text { Cath } \\
\text { bpm }\end{array}$ & $\begin{array}{c}\text { Pressure-volume } \\
\mathrm{s}\end{array}$ & $\begin{array}{c}\text { Contractility } \\
\mathrm{kPa}\end{array}$ & $\begin{array}{c}\text { Stroke work } \\
\mathrm{mJ}\end{array}$ & $\begin{array}{c}R_{\text {dist }} \\
\times 10^{8} \mathrm{~Pa} \times \mathrm{s}^{3} \mathrm{~m}^{3}\end{array}$ \\
\hline $\mathrm{Pt} \mathrm{\# 1}$ & 0.089 & 70 & 74 & 0.081 & 95 & 698 & 1.05 \\
$\mathrm{Pt} \mathrm{\# 2}$ & 0.165 & 83 & 61 & 0.142 & 85 & 853 & 0.52 \\
$\mathrm{Pt} \mathrm{\# 3}$ & 0.129 & 88 & 87 & 0.139 & 86 & 367 & 1.55 \\
$\mathrm{Pt} \mathrm{\# 4}$ & 0.133 & 81 & 59 & 0.098 & 100 & 893 & 0.85 \\
$\mathrm{Pt} \mathrm{\# 5}$ & 0.147 & 91 & 67 & 0.139 & 73 & 467 & 1.60 \\
$\mathrm{Pt} \mathrm{\# 6}$ & 0.163 & 82 & 75 & 0.120 & 73 & 462 & 1.40 \\
$\mathrm{Pt} \mathrm{\# 7}$ & 0.129 & 75 & 74 & 0.106 & 67 & 445 & 0.90 \\
$\mathrm{Pt} \mathrm{\# 8}$ & 0.096 & 74 & 95 & 0.161 & 56 & 304 & 1.10 \\
$\mathrm{Pt} \mathrm{\# 9}$ & 0.146 & 79 & 79 & 0.158 & 48 & 328 & 1.30 \\
$\mathrm{Pt} \mathrm{\# 10}$ & 0.129 & 49 & 75 & 0.086 & 80 & 907 & 0.54 \\
\hline
\end{tabular}

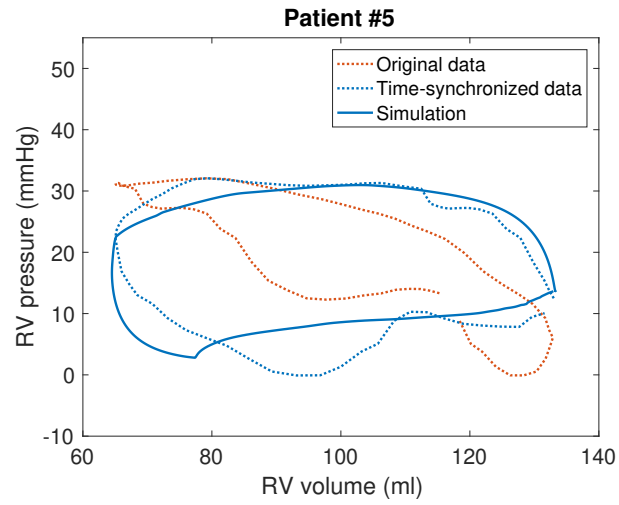

Fig. 7. Pressure-volume loops for right ventricle of patient \#5 with $11 \%$ and $30 \%$ of tricuspid and pulmonary regurgitation, respectively. 
box plot in Fig. 6 shows that in all 10 patients the shape of normalized timevarying elastance obtained from time-synchronized $\mathrm{P}-\mathrm{V}$ data is substantially closer to the experimentally measured curve [15]. This suggests that the timesynchronization restores the time-varying elastance to give a physiologically more meaningful relationship. Even though the time-varying elastance obtained in [15] was obtained in dogs, some data obtained in other animal species (e.g. pigs [13]) or even from invasive measurements in human patients [14,9] suggest their compatibility with [15]. Therefore, in the present work we assumed the normalized time-varying elastance [15] to be a physiological representative of ventricular pressure-volume relationship. The time offsets detected by the model appeared to vary among the patients (Table 1) demonstrating that the same offset cannot be applied to all patients even though the same setup of MR acquisition was used. The offsets between $\mathrm{P}-\mathrm{V}$ data were between 0.081 and $0.161 \mathrm{~s}$, what is in the order of the QRS duration in all patients in our study. In addition to that in patients $\# 2, \# 4, \# 5, \# 10$ the difference between MRI and pressure heart rates was more that $30 \%$. Therefore we hypothesize that when combining MRI and catherization techniques the offsets in the P-V data might be associated with several factors: wide QRS complex, difference in the heart rates, and an error in the order of temporal resolution of the MRI (0.020-0.030s).

The model employed in this work was a biomechanical model incorporating physiology of muscle contraction built on various spatial scales. Of note, any other heart model with adequate timings of P-V change could be used as a template in the proposed time-synchronization method. The advantage of using our model [3] is that it can also account for the valvular mechanics (inflow and/or outflow valve regurgitation, see [2]) and hence can be used for time-synchronization of the ventricles with more complicated physiology. Fig. 7 shows an example of model-assisted time-synchronization of the right ventricle of Patient \#5 with $11 \%$ and $30 \%$ of tricuspid and pulmonary regurgitation, respectively, where the absence of isovolumic phases would have limited the ability to yield a physiological P-V loop without using a model as a resynchronization template. In addition, the advantage of using a truly biophysical model is that it provides, as a "side effect", additional patient-specific mechanical indicators of various compartments of the cardiovascular system (some examples are displayed in Table 1). Those may not be directly visible in the data but are clinically relevant. For instance, myocardial contractility characterizes the functionality of the ventricle, the stroke work can provide information about myocardial energetics, and the distal resistance of the circulation system is an important indicator of a functional state or cardiovascular health in a variety of pathologies. The proposed time-synchronization strategy is not limited neither to the CMR \& catherization data acquisition protocol nor to the ventricular pressure \& volume data itself, and could be utilized in the variety of patients' data. It can be used e.g. to time-synchronize data acquired in an interventional CMR suite (iCMR, [11]). Moreover, the model can substitute e.g. a missing part of the cardiac cycle in the volume waveform (typically, in highly accelerated cine MRI in prospective ECG trigger). 


\section{Conclusion}

Time-synchronization of data using patient-specific biophysical modeling can be applied when combining various imaging techniques (echoardiography, dynamic $\mathrm{CT}$, or CMR) and/or when imaging is combined with catherization data. The proposed modeling framework is fast in computational time and can be employed in clinical settings allowing to plot high-quality P-V loops and extract some useful functional indices of the cardiovascular system.

\section{Acknowledgments}

The authors acknowledge the support of the Associated Team TOFMOD, created between Inria France and UT Southwestern Medical Center Dallas. The work was also funded in part by the W.B. \& Ellen Gordon Stuart Trust, The Communities Foundation of Texas and by the Pogue Family Distinguished Chair (awarded to Dr F. Gerald Greil in February, 2015). In addition, we would like to acknowledge Dr Philippe Moireau, Inria research team MEDISIM, for the development of the cardiac simulation software CardiacLab used in this work. Research reported in this publication was supported by Children's Health ${ }^{\mathrm{SM}}$ but the content is solely the responsibility of the authors and does not necessarily represent the official views of Children's Health ${ }^{\mathrm{SM}}$.

\section{References}

1. Caruel, M., Chabiniok, R., Moireau, P., Lecarpentier, Y., Chapelle, D.: Dimensional reductions of a cardiac model for effective validation and calibration. Biomechanics and Modeling in Mechanobiology 13(4), 897-914 (Aug 2014)

2. Chabiniok, R., Moireau, P., Kiesewetter, C., Hussain, T., Razavi, R., Chapelle, D.: Assessment of atrioventricular valve regurgitation using biomechanical cardiac modeling. In: International Conference on Functional Imaging and Modeling of the Heart. pp. 401-411. Springer (2017)

3. Chapelle, D., Le Tallec, P., Moireau, P., Sorine, M.: Energy-preserving muscle tissue model: formulation and compatible discretizations. International Journal for Multiscale Computational Engineering 10(2), 189-211 (2012)

4. Genet, M., Stoeck, C.T., Von Deuster, C., Lee, L.C., Kozerke, S.: Equilibrated warping: Finite element image registration with finite strain equilibrium gap regularization. Medical Image Analysis 50, 1-22 (Dec 2018)

5. Hill, A.V.: The heat of shortening and the dynamic constants of muscle. Proceedings of the Royal Society of London. Series B - Biological Sciences 126(843), 136-195 (Oct 1938)

6. Huxley, A.: Muscle structure and theories of contraction. Prog. Biophys. Biophys. Chem 7, 255-318 (1957), https://ci.nii.ac.jp/naid/10005175870/en/

7. Kimmig, F., Chapelle, D., Moireau, P.: Thermodynamic properties of muscle contraction models and associated discrete-time principles. Advanced Modeling and Simulation in Engineering Sciences 6(1), 1-36 (Dec 2019) 
8. Klotz, S., Hay, I., Dickstein, M.L., Yi, G.H., Wang, J., Maurer, M.S., Kass, D.A., Burkhoff, D.: Single-beat estimation of end-diastolic pressure-volume relationship: a novel method with potential for noninvasive application. American Journal of Physiology-Heart and Circulatory Physiology 291(1), H403-H412 (Jul 2006)

9. Le Gall, A., Vallée, F., Chapelle, D., Chabiniok, R.: Minimally-invasive estimation of patient-specific end-systolic elastance using a biomechanical heart model. In: FIMH 2019 - 10th Functional Imaging and Modelling of the Heart. Bordeaux, France (Jun 2019), https://hal.archives-ouvertes.fr/hal-02153611

10. Le Gall, A., Vallée, F., Pushparajah, K., Hussain, T., Mebazaa, A., Chapelle, D., Gayat, É., Chabiniok, R.: Monitoring of cardiovascular physiology augmented by a patient-specific biomechanical model during general anesthesia. A proof of concept study. PLoS ONE 15(5), e0232830 (May 2020)

11. Reddy, S.R.V., Arar, Y., Abou Zahr, R., Gooty, V., Hernandez, J., Potersnak, A., Douglas, P., Blair, Z., Greer, J.S., Roujol, S., et al.: Invasive cardiovascular magnetic resonance (icmr) for diagnostic right and left heart catheterization using an mr-conditional guidewire and passive visualization in congenital heart disease. Journal of Cardiovascular Magnetic Resonance 22(1), 1-11 (2020)

12. Sainte-Marie, J., Chapelle, D., Cimrman, R., Sorine, M.: Modeling and estimation of the cardiac electromechanical activity. Computers \& Structures 84(28), 17431759 (Nov 2006)

13. Seemann, F., Arvidsson, P., Nordlund, D., Kopic, S., Carlsson, M., Arheden, H., Heiberg, E.: Noninvasive quantification of pressure-volume loops from brachial pressure and cardiovascular magnetic resonance. Circulation: Cardiovascular Imaging 12(1), e008493 (2019)

14. Senzaki, H., Chen, C.H., Kass, D.A.: Single-beat estimation of end-systolic pressure-volume relation in humans: a new method with the potential for noninvasive application. Circulation 94(10), 2497-2506 (1996)

15. Suga, H., Sagawa, K., Shoukas, A.A.: Load Independence of the Instantaneous Pressure-Volume Ratio of the Canine Left Ventricle and Effects of Epinephrine and Heart Rate on the Ratio. Circulation Research 32(3), 314-322 (Mar 1973) 